\title{
Garcinia atroviridis - a review on phytochemicals and pharmacological properties
}

\begin{abstract}
Garcinia atroviridis or commonly named as 'Asam Gelugur' among locals is extensively used as flavouring agent to provide sour sensation. Apart from being used as flavouring agent, G. atroviridis is also used in many ways to promote health traditionally. Previous investigations of the plant reported many interesting potential of antioxidant, antimicrobial, antifungal, antiobesity and lipid metabolism, cytotoxicity, antiinflammatory, antimalarial and antinicotine stress activities of G. atroviridis. This article discusses on G. atroviridis in aspect of its chemical constituents and bioactivities; in vitro and in vivo as well the clinical study made on this plant despite the limited data available. It is also an effort to update G. atroviridis data on its phytochemical and pharmacological data of the plant in the recent 15 years.
\end{abstract}

Keyword: Garcinia atroviridis; Hydroxycitric acid; Antioxidant; Antimicrobial; Antiinflammatory; Cytotoxic; Antihyperlipidaemia 